\title{
A mejor condición física mejores resultados de una ley contrala obesidad Better fitness, better results of a law against obesity
}

*Patricio Solis-Urra, ${ }^{*}$ Nathalia Fernández-Cueto, **Rodrigo Nanjarí, *Tamara Huber-Pérez, *María Paz Cid-Arnes, *Natalia Zurita-Corvalán, *Fernando Rodríguez-Rodríguez, *Carlos Cristi-Montero

*Pontificia Universidad Católica de Valparaíso (Chile), **Universidad de las Américas (Chile)

Resumen. Introducción: La condición física (CF) es un importante predictor de salud en niños y adolescentes, sin embargo, hasta la fecha se desconoce su posible efecto «protector» frente a periodos críticos vinculados a riesgo de obesidad. Objetivo: Evaluar a través de un diseño longitudinal y ecológico la influencia de la CF y su interacción con la implantación de la «Ley Súper 8» sobre diversos parámetros de la composición corporal durante las vacaciones de fiestas patrias (VFP). Metodología: 110 escolares participaron inicialmente del estudio. Tras aplicar los criterios de exclusión, 48 escolares entre 10 y 13 años (52\% niñas) conformaron la muestra final. Se determinó la CF (cardiorrespiratoria y muscular) y la composición corporal (peso, índice de masa corporal [IMC], índice de masa triponderal [IMT], índice de masa grasa [IMG] \% grasa y \% músculo), durante septiembre del año 2015 (sin ley) y 2016 (con ley). Un modelo general lineal y pruebas t-student fueron realizadas, estableciendo como significativo p<0,05. Resultados: Los escolares que presentan un bajo nivel de CF modificaron negativamente su composición corporal (peso corporal, IMG, IMC Z-score y \% grasa) durante las VFP tras la implementación de la ley $(\mathrm{p}<0,05)$, mientras que en los escolares con un alto nivel de CF se observaron cambios favorables en el \% músculo, IMG e IMT $(\mathrm{p}<0,05)$. Conclusión: La CF interactúa positivamente con la «Ley Súper 8». Solo los escolares con buena CF previenen el aumento de diversas variables de la composición corporal vinculadas a obesidad durante las VFP.

Palabras claves: legislación, obesidad, sobrepeso, capacidad cardiorrespiratoria, fuerza muscular, vacaciones.

Abstract. Background: Fitness is an important predictor of health in children and adolescents; however, to date has not been demonstrated the protective effect against critical periods related to obesity. Objective: To evaluate the influence of fitness on body composition parameters during national holidays, comparing the 1-year effects with and without the implementation of the "Law Super 8», considering the fitness as a preventive and possible potential law enhancer. Method: Sample was composed by 48 schoolchildren between 10 and 13 years of age. Fitness (cardiorespiratory and muscular) and body composition (weight, body mass index [BMI], triponderal mass index [TMI], fat mass index [FMI], \% fat and \% muscle) were evaluated during two week of September 2015 (without law) and 2016 (with law). A general linear model and t-student test were used, setting the significant value at $\mathrm{p}<0.05$. Results: body composition (body weight, FMI, BMI Z-score and \% fat) of those children presenting low fitness level worsened during national holidays after implementation of law $(\mathrm{p}<0.05)$, while those with high fitness level showed favorable changes in $\%$ muscular, FMI, and TMI $(\mathrm{p}<0.05)$. Conclusion: Changes produced due to «Super 8 » law seem to affect positively just children who have high fitness level, preventing increase of variables of body composition related to obesity.

Keywords: legislation, obesity, overweight, physical fitness, muscle strength, holiday.

\section{Introducción}

La obesidad infantil es un problema de salud pública anivel mundial (Lobstein, Baur, \& Uauy, 2004; Ward et al., 2017). En la actualidad, la excesiva ingesta calórica y la disminución de la actividad física han generado un aumento sistemático de diversas variables de la composición corporal en niños y adolescentes, traduciéndose en un mayor riesgo de sufrir enfermedad cardiovascular en la adultez (Lobstein et al., 2004; WHO, 2016). Anivel mundial, al año 2016, el 18\% de los niños entre 5 y 18 años presentaron sobrepeso, mientras que el 6\% de los niños y el 8\% de las niñas presentaron algún grado de obesidad (WHO, 2016).

En Chile, según cifras de la Organización Mundial de la Salud (OMS) el 35,4\% de los niños entre cinco y 19 años presenta sobrepeso u obesidad (WHO, 2017), una cifra realmente desalentadora. En respuesta a esta importante problemática nacional (Garber, Sajuria, \& Lobelo, 2014), y de acuerdo con las recomendaciones de la OMS (WHO, 2016), en el año 2016 entró en vigencia la implementación de la ley 20.606, comúnmente llamada «Ley Súper 8». Esta ley, referida a la composición nutricional de los alimentos y su publicidad, tiene por objetivo: a) regular la venta de alimentos con un alto contenido de nutrientes críticos (grasas, azúcar y sal), b) regular su publicidad en menores de 14 años y c) promover la alimentación saludable en las escuelas del país (República de Chile. Ministerio de Salud, 2015). Debido a su reciente implementación, hasta la fecha se desconoce su posible impacto sobre la composición corporal de los escolares.

En otro contexto, recientemente ha sido postulada una hipótesis denominada SDH (por sus siglas en inglés: Structured Day Hypothesis) que expone que los días estructurados (por ejemplo, tiempo en las escuelas) podrían proteger a los niños de comportamientos

Fecha recepción: 18-07-18. Fecha de aceptación: 13-12-18 Carlos Cristi-Montero

carlos.cristi.montero@gmail.com «obesogénicos» en comparación a los días no estructurados (Brazendale et al., 2017). Ante esto, existe evidencia que demuestra un aumento anormal del peso corporal y modificación de la composición corporal en niños durante ciertos periodos críticos del año como lo son las vacaciones de verano (von Hippel, Powell, Downey, \& Rowland, 2007), fines de semana (Brazendale et al., 2017) y vacaciones de fiestas patrias (VFP) (Cristi-Montero et al., 2014; Cristi-Montero, et al., 2016). En Chile, las VFP han demostrado ser un importante periodo crítico, ya que los escolares modifican significativamente sus hábitos de alimentación y niveles de actividad física, provocando ganancias excesivas de peso y grasa corporal (Cristi-Montero et al., 2014; Cristi-Montero, et al., 2016). Este incremento, podría incluso mantenerse a lo largo del tiempo y sumarse al aumento de peso de otros periodos críticos, provocando un efecto de «tipo escalera» (Cristi-Montero et al., 2014; Cristi-Montero, et al., 2016; Cristi-Montero, 2017).

Por otra parte, tanto la capacidad cardiorrespiratoria como la fuerza muscular, ambos componentes de la condición física (CF) general, han demostrado influir de forma significativa en la salud de adultos (Campos, González-Víllora, Gómez, \& Martins, 2019; Cristi-Montero, Ramírez-Campillo, et al., 2016; Ramírez-Vélez et al., 2016) y niños (Ortega, Ruiz, Castillo, \& Sjöström, 2008). Un buen nivel de CF es capaz de atenuar los efectos negativos de algunas condiciones fisiopatológicas, tales como la insulino-resistencia y el riesgo cardiometabólico (Brouwer, Stolk, Liem, Lemmink, \& Corpeleijn, 2013; Nyström et al., 2017; Vásquez et al., 2017) e incluso contrarrestar los efectos negativos de la adiposidad sobre el rendimiento académico (García-Hermoso, Esteban-Cornejo, Olloquequi, \& Ramírez-Vélez, 2017). No obstante, la población escolar en Chile presenta una alta prevalencia de inactividad física y bajos niveles de CF, especialmente en niñas (Aguilar-Farías et al., 2016; Garber et al., 2014) de manera similar que otras poblaciones escolares (Viera, Quiñones, \& Araujo, 2018; Zurita-Ortega et al., 2018).

El objetivo del presente estudio fue evaluar la influencia de la CF sobre la variación de diversos parámetros de la composición corporal tras un conocido periodo crítico, y comparar su efecto entre un año sin 
y con la implementación de la «Ley Súper 8», considerando el nivel de CF de los escolares como un posible agente preventivo y potenciador de la ley.

\section{Material y Métodos}

\section{Diseño de estudio}

Las evaluaciones se realizaron en dos etapas. La primera, se llevó a cabo en el mes de septiembre del año 2015 (sin ley), y la segunda, en septiembre del año 2016 (con ley). Durante cada año se realizaron dos evaluaciones, una antes y otra tras las VFP. Cada evaluación se realizó entre las nueve y 11 a.m. Se les solicitó a los participantes asistir con pantalón deportivo y una camiseta a las evaluaciones. Siempre fueron medidos por el mismo evaluador, quien además, era del mismo sexo. El estudio se rigió bajo las normas deontológicas de la Declaración de Helsinki (World Medical Association., 2001).

Respecto a la implementación de la «Ley Súper 8», ésta comenzó a regir oficialmente en junio del 2016, no obstante, los establecimientos educacionales podían comenzar desde inicios del año escolar (marzo 2016) a implementar voluntariamente contenidos curriculares y actividades que fomentaran la educación en alimentación saludable y actividad física. El establecimiento estudiado incluyó contenidos en diversas asignaturas tales como educación física y ciencias naturales. En educación física se incluyeron temas vinculados a estilos de vida saludable, actividad física, nutrición y vida al aire libre, mientras que en ciencias naturales se incluyeron temas vinculados a factores de riesgo y alimentación saludable. Además, cerró el quiosco del establecimiento en donde se vendían golosinas y comida poco saludable, e implementó que los escolares llevaran sus colaciones desde sus hogares (frutas, yogurt, frutos secos, etc.).

\section{Participantes}

Los participantes fueron escolares de $5^{\text {to a }} 8^{\text {vo }}$ básico (entre 10 y 13 años) que pertenecían a un establecimiento particular subvencionado ubicado en la ciudad de Viña del Mar, Chile. Fueron incluidos aquellos niños y niñas, que tanto ellos como sus apoderados, firmaron voluntariamente el consentimiento o asentimiento informado de participación. La muestra inicial fue de 110 escolares, 23 de ellos fueron excluidos por no presentar algún dato en las variables principales de estudio (2016/ 2017); de la muestra restante, 30 escolares fueron retirados del establecimiento (año 2016) y nueve fueron excluidos por presentar problemas estomacales durante alguna de las semanas de evaluación (vómitos y/o diarrea). La muestra final fue de 48 escolares (23 niños y 25 niñas).

\section{Procedimientos}

\section{Composición corporal}

El peso se obtuvo utilizando una balanza -previamente calibradacon una precisión de 0,1 kg (Tanita ${ }^{\circledR}$ Japón, modelo HD-313) y la estatura fuemedida con un estadiómetro portátil (Seca ${ }^{\circledR}$ Alemania, modelo 216). Se determinó el estado nutricional según las recomendaciones de la OMS a través del IMC Z-score obtenido por el software WHO AnthroPlus ${ }^{\circledR}$. Además, se calculó el índice de masa triponderal (IMT), obtenido entre el cociente del peso corporal (kg) y la estatura al cubo $\left(\mathrm{m}^{3}\right)$, ya que este ha sido propuesto como un indicador más preciso que el IMC Z-score en adolescentes para determinar su masa grasa (Peterson et al., 2017). El porcentaje de masa grasa y masa muscular se evaluó a través de un sistema de bioimpedancia multifrecuencia octopolar (InBody modelo S10, Biospace, Seoul, Korea) siguiendo el protocolo propuesto por el Grupo Español de Cineantropometría (Alvero Cruz, 2009). Para realizar esta medición, previamente se les solicitó a los escolares que: a) no consumieran ningún tipo de alimento al menos dos horas antes de la prueba, b) no realizaran ejercicio físico extenuante que los pudiera deshidratar durante las 12 horas previas a la medición, c) haber orinado 30 minutos antes de la medición, y por último, d) no llevar elementos metálicos en el cuerpo (reloj, anillos, pulseras, pendientes, piercings, etc.). Con los resultados de la bioimpedancia se calculó el índice de masa grasa (IMG), el cuál es un importante indicador vincula- do a riesgo cardiovascular en adolescentes (Oliveira et al., 2015), este valor se obtuvo entre el cociente de la masa grasa $(\mathrm{kg})$ y la estatura al cuadrado $\left(\mathrm{m}^{2}\right)$.

\section{Condición física}

La fuerza muscular fue evaluada a través de la fuerza de prensión manual utilizando un dinamómetro digital marca Jamar ${ }^{\circledR}$ (con una precisión de 100 g). Se calculó la fuerza relativa, considerada como un indicador de capacidad muscular (Pacheco Herrera, Ramírez-Vélez \& Correa Bautista, 2016), dividiendo el valor máximo obtenido en la prueba de fuerza con el peso corporal de cada niño. Por otra parte, la capacidad cardiorrespiratoria $\left(\mathrm{VO}_{2 \max }\right.$ : consumo máximo de oxígeno) fue obtenida aplicando el test de Course Navette. La ecuación empleada fue: $\mathrm{VO}_{2 \max }$ : 31,025 + (3,238 x v) - (3,238 xe) + (v xe), donde v: velocidad máxima (km/h)y e: edad del sujeto (años) (Léger, Mercier, Gadoury, \& Lambert, 1988).

\section{Análisis estadístico}

Los valores son presentados como media \pm desviación estándar (DE). Todas las variables pasaron la prueba de normalidad de Kolmogorov-Smirnov $(p>0,05)$. Se realizó una prueba t-student para establecer diferencias entre niños y niñas. Se calculó el Z-score de cada niño (ajustado al sexo y edad), y de esta manera se dividió la muestra en dos grupos (mediana): alto y bajo nivel según cada variable de CF (capacidad cardiorrespiratoria, fuerza relativa y CF global). Por tanto, la CF global se calculó mediante la suma del Z-score de la CF cardiorrespiratoria y fuerza relativa.

Se aplicó un modelo lineal general dividido por grupo. Se utilizó como variable dependiente la variación ( $\Delta$ semana post - pre VFP) del parámetro vinculado a composición corporal (peso corporal, IMC Zscore, IMT, IMG \% grasa y \% músculo), y se compararon los valores dependiendo de si estaba o no implementada la ley (año 2015 vs. 2016). Además, se calculó el coeficiente de correlación de Pearson para establecer el grado de asociación de las variables entre un año y otro. Un valor de $p<0,05$ fue establecido como significativo estadísticamente. Todos los análisis se realizaron con el software IBM SPSS Statistics v 21.0 para MAC (IBM Corp, Armonk, New York, USA).

\section{Resultados}

La tabla 1 presenta las características de los participantes. No se observaron diferencias significativas de acuerdo al sexo.

\begin{tabular}{|c|c|c|c|c|}
\hline & Todos & Niñas & Niños & \\
\hline & $\mathrm{n}=48$ & $\mathrm{n}=25$ & $\mathrm{n}=23$ & $\mathrm{p}$-valor \\
\hline Edad (años) & $11,7 \pm 1,1$ & $11,7 \pm 1,1$ & $11,8 \pm 1,1$ & 0,65 \\
\hline Peso (kg) & $44,0 \pm 10,8$ & $44,2 \pm 9,6$ & $43,8 \pm 12,1$ & 0,90 \\
\hline Talla $(\mathrm{cm})$ & $148,6 \pm 10,3$ & $147,7 \pm 8,7$ & $149,6 \pm 12,0$ & 0,53 \\
\hline IMC Z-score (kg/m²) & $0,477 \pm 1,20$ & $0,524 \pm 1,08$ & $0,426 \pm 1,34$ & 0,78 \\
\hline Grasa (\%) & $23,5 \pm 6,9$ & $24,7 \pm 6,4$ & $22,1 \pm 7,4$ & 0,35 \\
\hline Músculo (\%) & $40,9 \pm 3,8$ & $40,3 \pm 3,6$ & $41,6 \pm 4,0$ & 0,39 \\
\hline IMT $\left(\mathrm{kg} / \mathrm{m}^{3}\right)$ & $10,2 \pm 3,1$ & $10,8 \pm 3,0$ & $9,5 \pm 3,3$ & 0,30 \\
\hline IMG $\left(\mathrm{kg} / \mathrm{m}^{2}\right)$ & $23,5 \pm 6,9$ & $24,7 \pm 6,4$ & $22,1 \pm 7,4$ & 0,35 \\
\hline Fuerza Relativa (kg/m) & $0,48 \pm 0,1$ & $0,45 \pm 0,0$ & $0,51 \pm 0,1$ & 0,35 \\
\hline $\mathrm{VO}_{2 \operatorname{MAX}}(\mathrm{ml} / \mathrm{kg} / \mathrm{min})$ & $43,2 \pm 2,9$ & $42,8 \pm 2,9$ & $43,6 \pm 2,9$ & 0,27 \\
\hline
\end{tabular}

\section{Capacidad cardiorrespiratoria}

La figura 1 presenta la variación de los marcadores de composición corporal de acuerdo a los distintos años de medición (2015 vs. 2016) y agrupados según nivel de capacidad cardiorrespiratoria (bajo o alto nivel de $\left.\mathrm{VO}_{2 \max }\right)$. En el grupo de alta capacidad cardiorrespiratoria no se apreciaron diferencias significativas en la variación entre cada año, excepto para el \% de masa muscular, donde se observó que en el año de aplicación de la ley la masa muscular disminuyó significativamente $(p<0,05)$. En el grupo de baja capacidad cardiorrespiratoria se observó un aumento significativo en el peso corporal, IMC Z-score y \% de masa grasa en el año con ley respecto al año sin la implementación de esta.

\section{Fuerza muscular}

La figura 2 presenta la variación de los marcadores de la composi- 

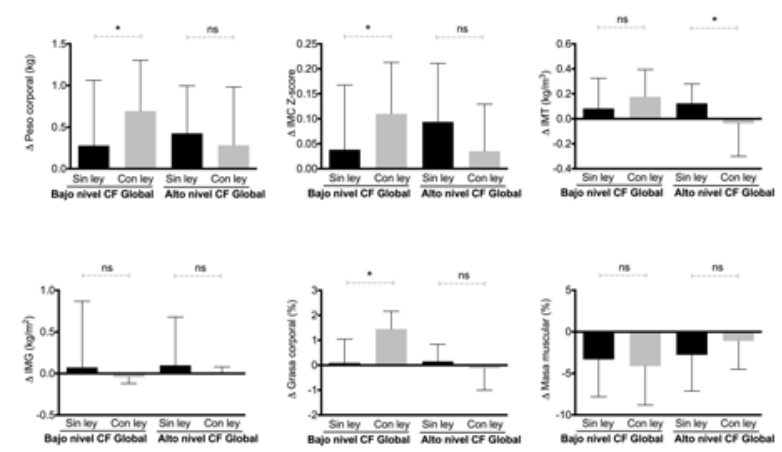

Figura 1. Comparación de diversos indicadores antropométricos según la implementación de la ley súper 8 y el nivel de condición física de los escolares: Capacidad cardiorrespiratoria $\left(\mathbf{V O}_{2 \max ) .} \mathrm{VO}_{2 \max }\right.$ : consumo de oxigeno máximo ajustado a la edad y sexo. Análisis ajustado a niveles de fuerza. IMC: Índice de Masa Corporal; IMT: Índice Masa Triponderal; IMG: Índice Masa Grasa. ns: sin significancia estadística; ${ }^{*} p<0,05$.
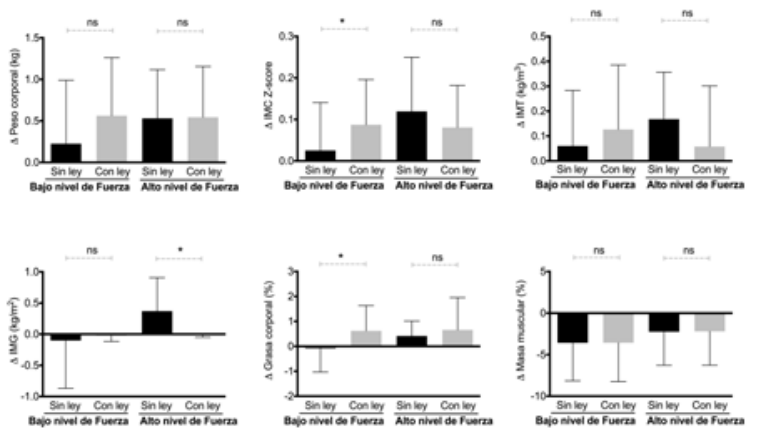

Figura 2. Comparación de diversos indicadores antropométricos según la implementación de la "Ley Súper 8" y el nivel de condición física de los escolares: Fuerza muscular relativa. Fuerz relativa ajustada a la edad y sexo. Análisis ajustado a niveles de $\mathrm{VO}_{2 \max }$. IMC: Índice de Masa Corporal; IMT: Índice Masa Triponderal; IMG: Índice Masa Grasa. ns: sin significancia estadística; * $p<0,05$
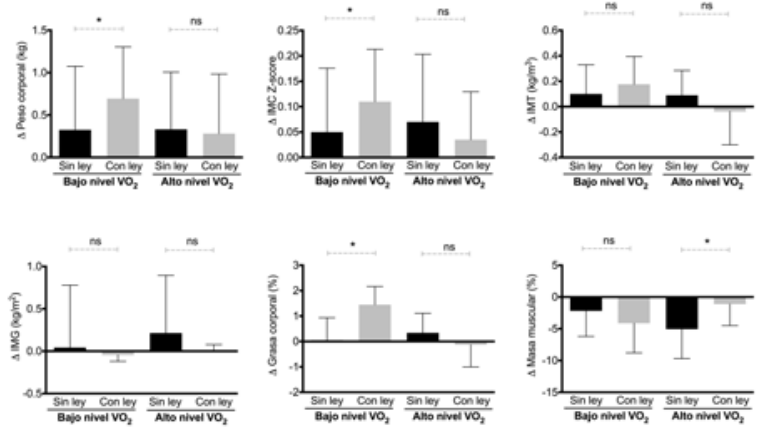

Figura 3. Comparación de diversos indicadores antropométricos según la implementación de la "Ley Súper 8" y el nivel de condición física de los escolares: Condición física global. Fitness global: condición física global (suma del Z-score $\mathrm{VO}_{2 \max } \mathrm{y} Z$-score fuerza relativa ajustado a la edad y sexo). IMC: Índice de Masa Corporal; IMT: Índice Masa Triponderal; IMG: Índice Masa edad y sexo). IMC: Indice de Masa Corporal; IMT:
Grasa. ns: sin significancia estadística; $* p<0,05$.
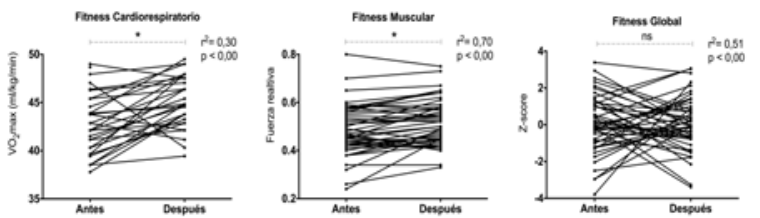

Figura 4. Variación y asociación de la condición física de los escolares de un año a otro.

ción corporal agrupados en relación al nivel de fuerza muscular relativa (bajo o alto nivel de fuerza relativa). En el grupo de alto nivel de fuerza muscular relativa se observó una diferencia significativa del IMG entre los años 2015 y $2016(p<0,05)$, mientras que el resto de los marcadores no presentaron diferencias. Quienes presentaron un bajo nivel de fuerza relativa aumentaron significativamente el IMC Z-score y \% de grasa $(p<0,05)$.

\section{Condición física global}

La figura 3 presenta la variación de los marcadores de composición corporal respecto al nivel de CF global (bajo o alto nivel de CF global). Quienes poseían un alto nivel de CG global presentaron diferencias significativas en el IMT, donde se aprecia una disminución significativa al incorporarse la ley. Por otra parte, quienes poseían un bajo nivel de
CF global presentaron diferencias significativas en el peso corporal, IMC Z-score y \% grasa corporal entre un año y otro, siendo el año con ley el que presentó niveles más elevados.

La figura 4 presenta el coeficiente de correlación de Pearson entre un año con y sin implementación de la ley graficando la variación intrasujeto para capacidad cardiorrespiratoria, fuerza relativa y CF global.

\section{Discusión}

El principal hallazgo del presente estudio sugiere que la implementación de la «Ley Súper 8» podría influir favorablemente sobre diversos parámetros vinculados a obesidad durante un periodo crítico como lo son las VFP en Chile, sin embargo, este efecto parecería afectar principalmente a escolares con buena CF.

A nuestro entender, este estudio es el primero en evaluar la influencia de la CF sobre la modificación de la composición corporal durante un periodo crítico. Previamente, ha sido descrito que durante las VFP los escolares chilenos tienden a aumentar entre 250 y 600 gramos su peso corporal (Cristi-Montero et al., 2014; Cristi-Montero, Munizaga, et al., 2016), no obstante, considerando nuestros resultados, esta variación podría depender del nivel de CF que posea el niño o niña. En adultos, se ha demostrado que poseer un alto nivel de CF tendría un efecto protector sobre la ganancia de peso (Tucker \& Peterson, 2010) mientras que, en niños, se ha observado que quienes presentan un alto nivel de CF tienen una menor ganancia de peso en comparación a sus pares con bajos niveles de CF, sin embargo, el periodo de vacaciones afectaría negativamente a su nivel de CF (Brazendale et al., 2017; Drenowatz et al., 2013).

Una posible explicación de estas diferencias se debería a que las personas con un bajo nivel de CF presentan un elevado nivel de sedentarismo y menor nivel de actividad física, y por tanto, un reducido gasto de energía (balance energético positivo) (Cristi-Montero, 2017). Otra explicación es que, durante estos días no estructurados, los niños tienden a comer más de lo requerido (Baranowski et al., 2014), generando así un desequilibrio entre la ingesta y el gasto energético. Este planteamiento fue corroborado en un estudio previo, donde se observó que los escolares aumentaron significativamente la ingesta calórica y mantuvieron o disminuyeron los niveles de actividad física durante las VFP (Cristi-Montero, et al., 2016). Cabe señalar que la variabilidad del peso corporal puede ser consecuencia de una deficiencia en los mecanismos responsables de la regulación del peso homeostático, siendo esta variabilidad un predictor de la ganancia de peso a largo plazo (Lowe, Feig, Winter, \& Stice, 2015) y por tanto, un factor de riesgo vinculado al desarrollo de obesidad en la adultez.

Por otro lado, recientemente se ha evidenciado que la «Ley Súper 8» tiene una aceptación favorable en los escolares chilenos (Olivares et al., 2016), pero hasta la fecha no se había evaluado su impacto sobre el estado nutricional de los niños. En consecuencia, con los resultados obtenidos, resulta imprescindible apoyar y reforzar esta ley con políticas gubernamentales enfocadas principalmente en aumentar los niveles de actividad física y ejercicio físico a nivel escolar, dirigidas principalmente a aumentar el tiempo dedicado a realizar ejercicio físico dentro de un rango de intensidad moderada-vigorosa (Cristi-Montero, RamírezCampillo, et al., 2016; Laguna, Ruiz, Lara, \& Aznar, 2013), con el fin de mejorar los niveles de CF de los niños y adolescentes.

Diversas estrategias se han establecido para mejorar la actividad física en las escuelas por ejemplo, implementar «recreos activos» de al menos 20 minutos cada día; incluir actividad física dentro de otras asignaturas, o establecer nuevos y mejores espacios para promover la práctica de actividad física al interior de los establecimientos, tanto antes, durante, como después de la jornada escolar, entre otras (Hills, Dengel, \& Lubans, 2015). No obstante, urge la necesidad de estudiar los factores que favorezcan y aseguren -de alguna manera- el éxito de este tipo de estrategias. Al respecto Bustos, Olivares, Leyton, Cano, \& Albala (2016) destacan que la actividad física en conjunto con intervenciones nutricionales, podrían mejorar los parámetros de obesidad infantil, pero que debe ser mejorada la efectividad de estas intervenciones. De 
esta forma, es plausible pensar -de acuerdo a nuestros resultados- que los escolares con un alto nivel de CF podrían ser quienes se vean más favorecidos con la implementación de estrategias que pretendan fomentar estilos de vida saludable y mejorar diversos parámetros vinculados a la composición corporal. No obstante, estudios de intervención, controlados y aleatorizados son necesarios para confirmar lo expuesto.

El presente estudio presenta ciertas limitaciones importantes de señalar. La primera, relacionada con el tamaño y selección de la muestra según disponibilidad, hace que nuestros resultados no puedan ser generalizados a toda la población, no obstante, los resultados obtenidos van en línea con resultados emanados en grandes poblaciones. Una segunda limitación se relaciona con la imposibilidad de contar con un análisis del tipo de alimentación y calorías consumidas durante este periodo crítico debido a los malos resultados obtenidos durante el pilotaje. A pesar de esto, determinamos que analizar las VFP independiente del consumo energético permite responder a nuestra pregunta de investigación con objetividad apoyándonos en la literatura.

Este estudio establece que los resultados esperados por la «Ley Súper 8» parecen ir en el camino correcto, no obstante, esta influiría principalmente en escolares con un alto nivel de CF, una minoría dentro de la realidad chilena (Aguilar-Farias et al., 2016; Garber et al., 2014). Por tanto, estos resultados pueden ayudar a tomar decisiones a nivel de país respecto a la implementación de nuevas estrategias para la promoción de estilos de vida saludable, las cuales integren no solo hábitos de alimentación, sino también potenciar y crear estrategias para aumentar los niveles de CF de la población escolar con el fin de prevenir comorbilidades en el futuro.

\section{Conclusiones}

Presentar un elevado nivel de CF podría atenuar el efecto negativo que genera un periodo crítico sobre diversos indicadores vinculados a obesidad en escolares y, además podría jugar un importante rol potenciando los resultados esperados tras la implementación de la «Ley Súper 8». Futuros estudios deberían considerar la CF de los escolares al investigar la influencia de distintos periodos críticos vinculados a la variación de la composición corporal durante el año.

\section{Agradecimientos}

Agradecemos al director del Colegio Diego Velásquez (Santa Julia, Viña del Mar), Sr. Julio Molina Araos, por su disposición a apoyarnos en este estudio, y en especial al Jefe de Unidad Técnica Pedagógica, Sr. Juan Luis Carreras, por su invalorable apoyo durante todo el transcurso del proyecto. Sin duda, su apoyo demuestra el interés que tiene el Colegio en mejorar el estado de salud de sus escolares. Agradecemos también, a todos los que ayudaron en la recolección de datos, Srtas. Javiera Meneses, Nataly Godoy, Carolina Cruz, María Cecilia Malebrán y Sres. Javier Calderón, Javier Sánchez y Jorge Olivares.

\section{Referencias}

Aguilar-Farias, N., Cortinez-O’Ryan, A., Sadarangani, K. P., Von Oetinger, A., Leppe, J., Valladares, M., ... Cristi-Montero, C. (2016). Results From Chile's 2016 Report Card on Physical Activity for Children and Youth. Journal of Physical Activity and Health, 13(11 Suppl 2), S117-S123. https://doi.org/10.1123/jpah.20160314

Alvero Cruz, J. E. Al. (2009). Protocolo de valoración de la composición corporal para el reconocimiento médico-deportivo. Documento de Consenso del Grupo Español de Cineantropometría de la Federacion Española de Medicina del Deporte. Archivos de Medicina Del Deporte, 26(Cc), 166-179. Retrieved from http://femede.es/ documentos/ConsensoCine131.pdf

Baranowski, T., O’Connor, T., Johnston, C., Hughes, S., Moreno, J., Chen, T.-A., . . . Baranowski, J. (2014). School Year Versus Summer Differences in Child Weight Gain:ANarrative Review. Childhood
Obesity, 10(1), 18-24. https://doi.org/10.1089/chi.2013.0116

Brazendale, K., Beets, M. W., Weaver, R. G., Pate, R. R., TurnerMcGrievy, G. M., Kaczynski, A. T., ... von Hippel, P. T. (2017). Understanding differences between summer vs. school obesogenic behaviors of children: The structured days hypothesis. International Journal of Behavioral Nutrition and Physical Activity, 14(1), 1-14. https://doi.org/10.1186/s12966-017-0555-2

Brouwer, S. I., Stolk, R. P., Liem, E. T., Lemmink, K. A. P. M., \& Corpeleijn, E. (2013). The role of fitness in the association between fatness and cardiometabolic risk from childhood to adolescence. Pediatric Diabetes, 14(1), 57-65. https://doi.org/10.1111/j.13995448.2012.00893.x

Bustos, N., Olivares, S., Leyton, B., Cano, M., \& Albala, C. (2016). Impact of a school-based intervention on nutritional education and physical activity in primary public schools in Chile (KIND) programme study protocol: Cluster randomised controlled trial. BMC Public Health, 16(1), 1217. https://doi.org/10.1186/s12889016-3878-z

Campos, F., González-Víllora, S., Gómez, D. G, \& Martins, F. M. L. (2019). Benefits of 8-week fitness programs on health and fitness parameters (Beneficios de los programas de fitness de ocho semanas en parámetros de salud y fitness). Retos(35), 224-228.

Cristi-Montero, C. (2017). iVacaciones a la vista! Fundamentos y consideraciones para prevenir el aumento de peso corporal en escolares. Revista Médica de Chile, 145(1), 136-138. https://doi.org/ 10.4067/S0034-98872017000100020

Cristi-Montero, C., Bresciani, G, Alvarez, A., Arriagada, V., Beneventi, A., Canepa, V., ... Rodriguez-Rodriguez, F. (2014). Critical periods in the variation in body composition in school children. Nutricioin Hospitalaria, 30(4), 782-786. https://doi.org/10.3305/ nh.2014.30.4.7694

Cristi-Montero, C., Munizaga, C., Tejos, C., Ayala, R., Henriquez, R., Solis-Urra, P., ... Rodríguez-Rodríguez, F. (2016). Variations of body composition, physical activity and caloric intake in schoolchildren during national holidays. Eating and Weight Disorders/: EWD, 21(2), 251-255. https://doi.org/10.1007/s40519015-0229-5

Cristi-Montero, C., Ramírez-Campillo, R., Alvarez, C., Méndez, A. G, Martínez, M. A., Martínez, X. D., ... Celis-Morales, C. (2016). Fitness cardiorrespiratorio se asocia a una mejora en marcadores metabólicos en adultos chilenos. Revista Medica de Chile, 144(8), 980-989. https://doi.org/10.4067/S0034-98872016000800004

Drenowatz, C., Kobel, S., Kettner, S., Kesztyüs, D., Wirt, T., Dreyhaupt, J., \& Steinacker, J. M. (2013). Correlates of weight gain in German children attending elementary school. Preventive Medicine, 57(4), 310-314. https://doi.org/10.1016/j.ypmed.2013.06.004

Garber, M. D., Sajuria, M., \& Lobelo, F. (2014). Geographical variation in health-related physical fitness and body composition among chilean 8th graders:Anationally representative cross-sectional study. PLoS ONE, 9(9), e108053. https://doi.org/10.1371/ journal.pone.0108053

García-Hermoso, A., Esteban-Cornejo, I., Olloquequi, J., \& RamírezVélez, R. (2017). Cardiorespiratory Fitness and Muscular Strength as Mediators of the Influence of Fatness on Academic Achievement. Journal of Pediatrics, 187, 127-133.e3. https://doi.org/10.1016/ j.jpeds.2017.04.037

Hills, A. P., Dengel, D. R., \& Lubans, D. R. (2015). Supporting Public Health Priorities: Recommendations for Physical Education and Physical Activity Promotion in Schools. Progress in Cardiovascular Diseases, 57(4), 368-374. https://doi.org/10.1016/ j.pcad.2014.09.010

Laguna, M., Ruiz, J. R., Lara, M.T., \& Aznar, S. (2013). Recommended levels of physical activity to avoid adiposity in Spanish children. Pediatric Obesity, 8(1), 62-69. https://doi.org/10.1111/j.20476310.2012.00086.x

Léger, L. A., Mercier, D., Gadoury, C., \& Lambert, J. (1988). The multistage 20 metre shuttle run test for aerobic fitness. Journal of 
Sports Sciences, 6(2), 93-101. https://doi.org/10.1080/ 02640418808729800

Lobstein, T., Baur, L., \& Uauy, R. (2004). Obesity in children and young people: a crisis in public health. Obesity Reviews, 5(s1), 485. https://doi.org/10.1111/j.1467-789X.2004.00133.x

Lowe, M. R., Feig, E. H., Winter, S. R., \& Stice, E. (2015). Short-term variability in body weight predicts long-term weight gain. American Journal of Clinical Nutrition, 102(5), 995-999. https://doi.org/ 10.3945/ajcn.115.115402

Nyström, C. D., Henriksson, P., Martínez-Vizcaíno, V., Medrano, M., Cadenas-Sanchez, C., Arias-Palencia, N. M., ... Ortega, F. B. (2017). Does cardiorespiratory fitness attenuate the adverse effects of severe/morbid obesity on cardiometabolic risk and insulin resistance in children? A pooled analysis. Diabetes Care, 40(11), 1580-1587.https://doi.org/10.2337/dc17-1334

Olivares, S., Araneda, J., Morales, G., Leyton, B., Bustos, N., Hernández, M. A., \& Oyarzún, M. T. (2016). Actitudes de escolares chilenos de distinto nivel socioeconómico al inicio de la implementación de la ley que regula la venta y publicidad de alimentos altos en nutrientes críticos. Nutr. Hosp., 34, 431-438.

Oliveira, P. M. de, Silva, F.A. da, Souza Oliveira, R. M., Mendes, L. L., Netto, M. P., \& Cândido, A. P. C. (2015). Association between fat mass index and fat-free mass index values and cardiovascular risk in adolescents. Revista Paulista de Pediatria, pp. 30-37. https:// doi.org/10.1016/j.rpped.2015.06.003

Ortega, F. B., Ruiz, J. R., Castillo, M. J., \& Sjöström, M. (2008). Physical fitness in childhood and adolescence: a powerful marker of health. International Journal of Obesity, 32(1), 1-11. https:// doi.org/10.1038/sj.ijo.0803774

Pacheco-Herrera, J. D., Ramírez-Vélez, R., \& Correa-Bautista, J. E. (2016).Índice general de fuerza y adiposidad como medida de la condición física relacionada con la salud en niños y adolescentes de Bogotá, Colombia: Estudio FUPRECOL. Nutrición Hospitalaria, 33(3), 556-564. https://doi.org/10.20960/nh.261

Peterson, C. M., Su, H., Thomas, D. M., Heo, M., Golnabi, A. H., Pietrobelli, A., \& Heymsfield, S. B. (2017). Tri-Ponderal Mass Index vs Body Mass Index in Estimating Body Fat During Adolescence. JAMAPediatrics, 171(7), 629. https://doi.org/10.1001/ jamapediatrics.2017.0460

Ramírez-Vélez, R., Correa-Bautista, J. E., Lobelo, F., Izquierdo, M., Alonso-Martínez,A., Rodríguez-Rodríguez, F., \& Cristi-Montero, C. (2016). High muscular fitness has a powerful protective cardiometabolic effect in adults: influence of weight status. BMC Public Health, 16(1), 1012. https://doi.org/10.1186/s12889-016-
3678-5

República de Chile. Ministerio de Salud. Ley 20869 Sobre Publicidad de los Alimentos (2015). Santiago: Diario Oficial de Chile.

Tucker, L., \& Peterson, T. (2010). Fitness Level and Risk of Weight Gain in Middle-Age Women:A Prospective Cohort Study. Journal of Physical Activity and Health, 7(3), 308-315. https://doi.org/ 10.1123/jpah.7.3.308

Vasquez, F., Diaz, E., Lera, L., Meza, J., Curi, K., Torres, J., \& Burrows, R. (2017). Physical fitness and insulin sensitivity in a group of obese children from 8 to 13 years of age by puberal state. NUTRICION HOSPITALARIA,34(4), 808-813. https://doi.org/10.20960/ nh. 61

Viera, E. C., Quiñones, I. T., \& Araujo, J. A. G Relación entre actividad física, alimentación y familia en edad escolar (Relationship between physical activity, nutrition, and family in school-aged youth). Retos(34), 85-88

von Hippel, P. T., Powell, B., Downey, D. B., \& Rowland, N. J. (2007). The Effect of School on Overweight in Childhood: Gain in Body Mass Index During the School Year and During Summer Vacation. American Journal of Public Health, 97(4), 696-702. https://doi.org/ 10.2105/AJPH.2005.080754

Ward, Z. J., Long, M. W., Resch, S. C., Giles, C. M., Cradock, A. L., \& Gortmaker, S. L. (2017). Simulation of Growth Trajectories of Childhood Obesity into Adulthood. New England Journal of Medicine, 377(22), 2145-2153. https://doi.org/10.1056/ NEJMoa1703860

WHO. (2016). Obesity and Overweight. Retrieved July 5, 2018, from http://www.who.int/news-room/fact-sheets/detail/obesity-andoverweight

WHO. (2017). Report of the Commission on Ending Childhood Obesity: implementation plan: executive summary. WHO. World Health Organization. Retrieved from http://www.who.int/end-childhoodobesity/publications/echo-plan-executive-summary/en/

World Medical Association., W. M. (2001). World medical association declaration of helsinki: Ethical principles for medical research involving human subjects. Bulletin of the World Health Organization. World Health Organization. Retrieved from http:// www.ncbi.nlm.nih.gov/pubmed/11357217

Zurita-Ortega, F., Ubago-Jiménez, J. L., Puertas-Molero, P., GonzálezValero, G., Castro-Sánchez, M., \& Chacón-Cuberos, R. Niveles de actividad física en alumnado de Educación Primaria de la provincia de Granada (Physical activity levels of Primary Education students in Granada). Retos(34), 218-221.

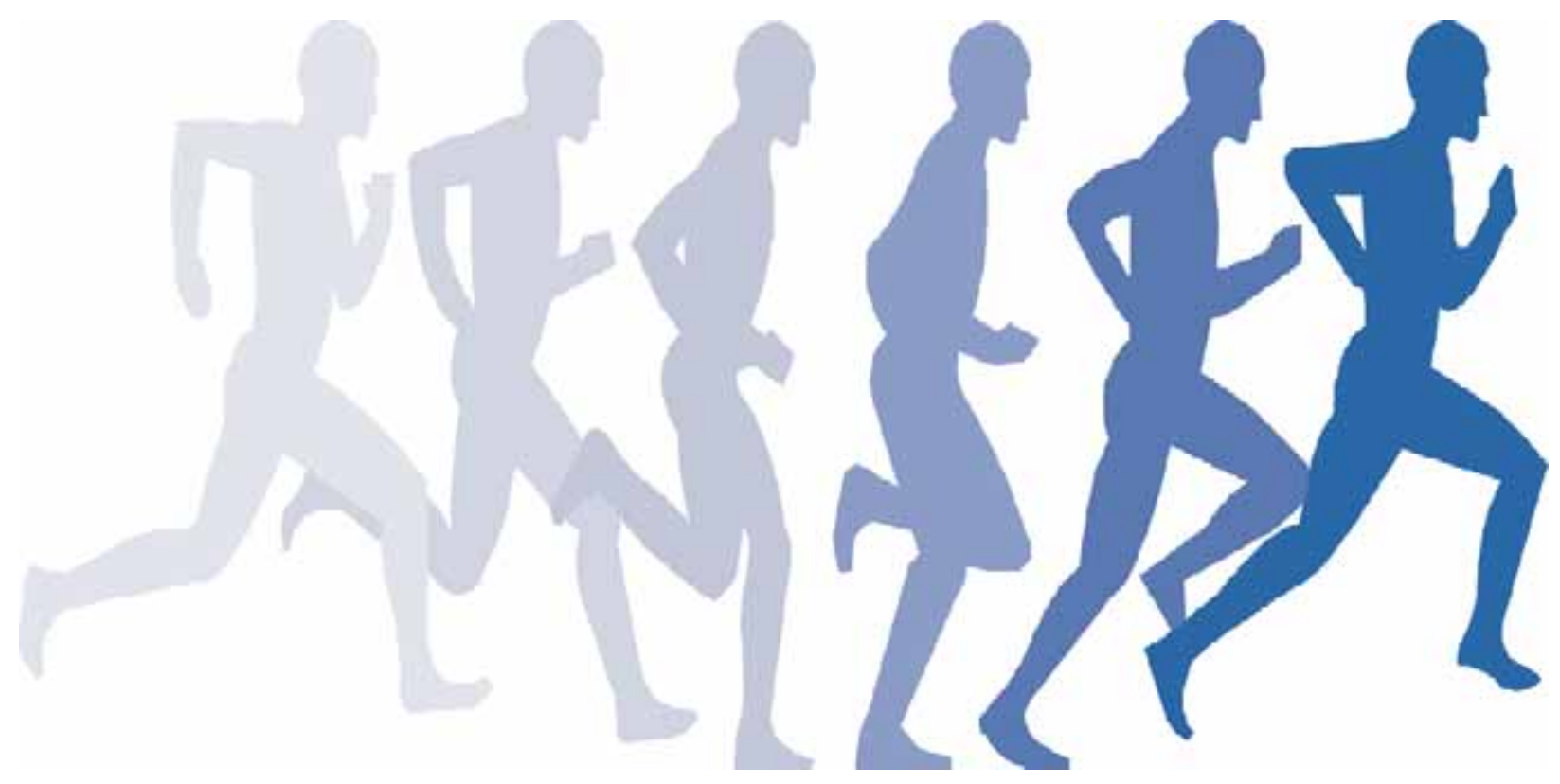

